\title{
Zusammenhang von arbeitsbezogenen psychischen Beanspruchungsfolgen mit subjektiver Schlafqualität und individueller Tagesschläfrigkeit*
}

\section{Associations of Work-Related Strain with Subjective Sleep Quality and Individual Daytime Sleepiness*}

Autoren

Robin Sebastian Schierholz, Sabine Darius, Irina Böckelmann

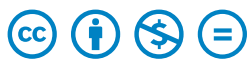

Institut

Bereich Arbeitsmedizin, Medizinische Fakultät, Otto-von-Guericke-Universität Magdeburg, Magdeburg

Schlüsselwörter

Schlaf, Stress, Arbeit, Psyche

Key words

sleep, stress, work, psyche

Bibliografie

DOI https://doi.org/10.1055/a-0873-7196

Online-Publikation: 8.5.2019

Dtsch Med Wochenschr 2019; 144: e121-e129

(c) Georg Thieme Verlag KG, Stuttgart · New York

ISSN 0012-0472

Korrespondenzadresse

Robin Sebastian Schierholz

Otto-von-Guericke-Universität Magdeburg

Medizinische Fakultät

Bereich Arbeitsmedizin, Leipziger Straße 44 (Haus 20), 39120 Magdeburg, Germany

iam@med.ovgu.de

\section{ZUSAMMENFASSUNG}

Einleitung Psychische Belastungen am Arbeitsplatz bergen ein hohes Gefährdungspotenzial für Gesundheit und Lebensqualität. Das Ziel dieser Studie war, die Zusammenhänge zwischen Beanspruchungsfolgen psychischer Belastung und subjektiver Schlafqualität sowie individueller Tagesschläfrigkeit zu untersuchen.

Methoden Es wurde eine Querschnittsuntersuchung an 84 einbezogenen Probanden durchgeführt. Arbeitsbezogene Beanspruchungsfolgen wurden mit den 3 Skalen Kognitive Irritation (KI), Emotionale Irritation (EI) und Gesamtindex (GI) der Irritations-Skala zur Erfassung arbeitsbezogener Beanspruchungsfolgen (IS), Schlafqualität mit dem Pittsburgh Sleep Quality Index (PSQI) und Tagesschläfrigkeit mit der Epworth Sleepiness Scale (ESS) evaluiert.

* Teil der Promotionsarbeit von Robin S. Schierholz (Part of the doctoral thesis by Robin S. Schierholz).
Ergebnisse Hinsichtlich der erfassten soziodemografischen und medizinischen Daten unterschieden sich die Probanden mit guter nicht von den Probanden mit schlechter Schlafqualität. In allen 3 Skalen der IS und in der ESS erreichten die schlechten Schläfer ungünstig höhere Werte als die guten Schläfer, wobei der Unterschied in Bezug auf die ESS nicht signifikant war. Höhere Punktzahlen in allen 3 Skalen der IS korrelierten mit höheren Werten im PSQI, jedoch lediglich höhere Punktzahlen in der El aus der IS auch mit höheren Punktzahlen in der ESS. Diskussion Arbeitsbezogene Beanspruchungsfolgen und subjektive Schlafqualität stehen in einer Wechselbeziehung, der in der heutigen Arbeitswelt eine wichtige Bedeutung zukommt. Wichtige Gesichtspunkte sind hierbei berufliche Unsicherheit und ständige Erreichbarkeit.

\section{ABSTRACT}

Introduction Mental strain at the work place involves high potential hazard for health and quality of life. The aim of this study was to examine the relationships between strain consequences of psychological strain and subjective sleep quality as well as daytime sleepiness.

Methods A cross-sectional study with 84 included participants was conducted. Work-related strain consequences were evaluated by means of the 3 scales Cognitive Irritation (CI), Emotional Irritation (EI) and Global Irritation (GI) of the Irritation Scale for Assessing Work-Related Strain Consequences (IS), sleep quality was assessed using the Pittsburgh Sleep Quality Index (PSQI) and daytime sleepiness was measured with the Epworth Sleepiness Scale (ESS).

Results Regarding the assessed socio-demographical and medical data subjects with good sleep quality did not differ from subjects with poor sleep quality. In all 3 scales of the IS and in the ESS poor sleepers reached unfavourable higher scores, but the difference with respect to the ESS was not significant. Higher scores in all 3 scales of the IS correlated with higher PSQI scores but only higher scores in the El of the IS correlated with higher ESS scores as well.

Discussion, Conclusions Work-related strain consequences and subjective sleep quality have an interrelationship with great importance in today's world of work. Considerable aspects here are job insecurity and constant availability. 


\section{Einleitung}

Psychische Belastungen am Arbeitsplatz zeigen sich als immer größer werdende Gesundheitsgefährdung [1]. Die Anzahl der Fehlzeiten aufgrund psychischer Störungen ist im Vergleich zu anderen Erkrankungen sehr stark angestiegen [2].

Gemäß DINEN ISO 10075 - 1 ist psychische Belastung „die Gesamtheit aller erfassbaren Einflüsse, die von außen auf den Menschen zukommen und psychisch auf ihn einwirken“ [3], während psychische Beanspruchung „die unmittelbare (nicht langfristige) Auswirkung der psychischen Belastung im Individuum in Abhängigkeit von seinen jeweiligen überdauernden und augenblicklichen Voraussetzungen, einschließlich der individuellen Bewältigungsstrategien“ [3], ist. Das Resultat psychischer Belastung kann eine positive Anregungswirkung, aber auch eine negative Beeinträchtigungswirkung sein. Wenn die Leistungsvoraussetzungen von Beschäftigten bei ihrer Arbeit qualitativ oder quantitativ über- oder unterschritten werden, kann es zu psychischer Fehlbeanspruchung kommen [3, 4]. So können kurz andauernde Auswirkungen von psychischer Beanspruchung einerseits Aktivierung nach sich ziehen, andererseits Ermüdung und ermüdungsähnliche Zustände wie psychische Sättigung und Monotonie oder Stress. Langzeitige Auswirkungen psychischer Beanspruchung können sowohl positive Beanspruchungsfolgen wie z. B. Weiterentwicklung der persönlichen und beruflichen Voraussetzungen, Übung und Verbesserung des Wohlbefindens des Beschäftigten nach sich ziehen, aber auch negative Beanspruchungsfolgen wie z. B. Burnout, psychosomatische Störungen und Frühverrentung [5].

Stress und psychische Belastungen bedeuten eine vermehrte psychische und physiologische Aktivierung [6]. Im Gegensatz dazu steht die psychologische und physiologische Verringerung der Aktivierung als Kennzeichen von Schlaf. Dabei spielt erholsamer Schlaf eine wichtige Rolle für psychisches Wohlbefinden und Gesundheit [7]. Es konnte ein Zusammenhang zwischen vermehrtem arbeitsbedingtem psychosozialem Stress und schlechter Schlafqualität in Querschnittsstudien [8, 9] und prospektiven Studien $[10,11]$ festgestellt werden. Auch sind letztlich aus psychischer Fehlbeanspruchung resultierende psychische Erkrankungen mit schlechter Schlafqualität assoziiert [12]. Vor diesem wissenschaftlichen Hintergrund ergab sich eine Fragestellung, der diese Studie nachging. Ziel dieser Studie war, die Zusammenhänge zwischen arbeitsbezogenen Beanspruchungsfolgen und subjektiver Schlafqualität sowie individueller Tagesschläfrigkeit zu untersuchen. Folgende Hypothesen wurden formuliert: (1) Schlechte Schläfer haben eine erhöhte Tagesschläfrigkeit; (2) Erniedrigte Schlafqualität geht einher mit erhöhter Tagesschläfrigkeit; (3) Schlechte Schlafqualität und erhöhte Tagesschläfrigkeit hängen mit erhöhter arbeitsbezogener Beanspruchung zusammen.

\section{Material und Methoden}

\section{Probanden und Studienaufbau}

Die Studienteilnehmer wurden über die arbeitsmedizinische Ambulanz des Bereichs Arbeitsmedizin der Medizinischen Fakultät der Otto-von-Guericke-Universität Magdeburg im Rahmen regulärer Vorsorgeuntersuchungen sowie über Bekanntgabe an Gesundheitstagen und Werbung unter lokalen Studierenden rekrutiert. Die Probanden erhielten den Fragebogenkatalog in Deutsch in der hiesigen arbeitsmedizinischen Ambulanz, füllten diesen zu Hause aus und erhielten nach dessen Rückgabe ein Feedback. Alle Probanden mit einer Ausnahme waren Muttersprachler. Folgende Ausschlusskriterien für die Teilnahme an der Studie wurden festgelegt: Schichtarbeit, regelmäßige Medikamenteneinnahme mit Einfluss auf den Herzrhythmus, Diabetes mellitus, unbehandelte Schilddrüsenerkrankungen und behandelte Schilddrüsenerkrankungen mit Schilddrüsenblutwerten außerhalb des Normbereichs, kardiale Erkrankungen, nächtliche Sauerstoffapplikation oder unterstützende maschinelle Ventilationstherapie sowie vorbestehende Erkrankungen des zentralen oder peripheren Nervensystems. Es resultierte daraus eine Gesamtstichprobe von 84 Probanden unterschiedlicher Berufe. Die Ermittlung der Stichprobengröße erfolgte anhand des $95 \%$ Konfidenzniveaus und der Fehlerspanne von $5 \%$. Vor Studienteilnahme bestätigten alle Probanden ihr Einverständnis schriftlich. Die Anonymität im Zusammenhang mit dem Datenschutz wurde sichergestellt. Die Registrierung der Studie erfolgte bei der Ethikkommission (Registriernummer 50/16) der Medizinischen Fakultät am 11.04.2016. Die Studie erhielt die zustimmende Bewertung der Ethikkommission der Otto-von-Guericke-Universität Magdeburg (Zustimmung der Ethikkommission vom 11.05.2016). Der Ethikkommission lagen das Studienprotokoll, das Formular für die Einverständniserklärung und das Informationsblatt für die Studienteilnehmer vor. Der experimentelle Teil der Studie erfolgte bis Oktober 2017. Die Probanden bekamen jeweils eine sechsstellige Probandennummer (515XXX).

Die Datensammlung und die Auswertungsgespräche erfolgten durch den Erstautor dieser Publikation, was dazu führte, dass die Fragebögen präzise auf Vollständigkeit geprüft wurden und somit bis auf bei zwei Fragebögen (siehe Abschnitt Arbeitsbezogene Beanspruchungsfolgen) keine fehlenden Daten entstanden.

\section{Fragebögen}

\section{Soziodemografische und medizinische Daten}

Im ersten Teil des Fragebogens sollten die Probanden Angaben zu Alter, Größe und Körpergewicht (zur Berechnung des Body-MassIndex (BMI) [13]), Taillen- und Hüftumfang (zur Berechnung des Taille-Hüft-Verhältnisses (THV), Messung des THV gemäß WHO [14]), sportlicher Aktivität, Tätigkeitsprofil und Rauchgewohnheiten inklusive der täglich konsumierten Zigaretten und Zahl der Raucherjahre machen. Außerdem wurde der arterielle Blutdruck (systolischer Blutdruck (RR sys) und diastolischer Blutdruck (RR dias)) nach einer 3- bis 5-minütigen Ruhephase gemessen. 


\section{Pittsburgh Sleep Quality Index (PSQI)}

Der PSQI [15] ist ein Selbstbeurteilungsfragebogen zur Erfassung der subjektiven Schlafqualität des letzten Monats. Er besteht aus 19 Fragen zur Selbsteinschätzung und 5 Fragen zur Fremdeinschätzung, welche nicht in die quantitative Auswertung eingehen. Die 19 Selbstbeurteilungsfragen generieren 7 KomponentenPunktzahlen (Subjektive Schlafqualität, Schlaflatenz, Schlafdauer, Schlafeffizienz, Schlafstörungen, Schlafmittelkonsum und Tagesschläfrigkeit). Die Summe der Komponenten-Punktzahlen ergibt die Gesamt-Punktzahl, wobei höhere Werte auf eine verminderte Schlafqualität hindeuten. Der von Buysse et al. [15] empfohlene Cut-off-Wert zur Unterscheidung zwischen guten und schlechten Schläfern liegt bei > 5 Punkten.

Mithilfe des erreichten PSQI-Resultats wurden die Probanden ( $n=84)$ in die zwei Gruppen „Gute Schläfer" ( $n=53)$ und „Schlechte Schläfer“ ( $n=31$ ) aufgeteilt, was die Grundlage für die Mittelwertvergleiche und die Zusammenhangsanalysen zwischen arbeitsbezogenen Beanspruchungsfolgen und Tagesschläfrigkeit bildete. Somit war auch die Vergleichbarkeit der Messmethoden als ein Punkt des STROBE-Statements [16] gegeben.

\section{Epworth Sleepiness Scale (ESS)}

Die ESS [17] ist ein Selbsteinschätzungsfragebogen zur Erfassung von Tagesschläfrigkeit in der letzten Zeit. Sie besteht aus 8 Beschreibungen unterschiedlich stark einschläfernder alltäglicher Situationen. Höhere Werte der Gesamtpunktzahl signalisieren eine höhere Tagesschläfrigkeit. Der bestimmte Cutoff-Wert der Gesamtpunktzahl für überhöhte Tagesschläfrigkeit liegt bei > 10 Punkten [17-20].

\section{Irritations-Skala zur Erfassung arbeitsbezogener Beanspruchungsfolgen (IS)}

Die IS [21] ist ein Selbsteinschätzungsfragebogen aus 8 Items, um psychische Beanspruchungsfolgen im arbeitsbezogenen Kontext zu beurteilen. Irritation wird dabei als Status psychischer Befindensbeeinträchtigung aufgrund erlebter Zieldiskrepanz mit Ruminationen entsprechend vermehrter Zielerreichungsbemühungen (Kognitive Irritation [KI]) und Gereiztheitsreaktionen entsprechend Zielabwehrtendenzen (Emotionale Irritation [EI]) definiert [22]. KI ist mehr mit tätigkeitsspezifischen Stressoren verknüpft, El dagegen vor allem mit sozialen Stressoren. Der Gesamtindex (GI) ist die Summe beider Einzelskalen. Höhere Werte zeigen eine höhere Ausprägung an Irritation.

\section{Statistik}

Im Rahmen der deskriptiven Statistik wurden Mittelwerte (MW) und Standardabweichungen (SD) sowie Mediane und Spannweiten (Range) berechnet. Um auf Normalverteilung zu prüfen, wurde der Kolmogorov-Smirnov-Anpassungstest verwendet. Im Falle einer Normalverteilung und intervallskalierten Daten wurde der Zweistichproben-t-Test für unabhängige Stichproben eingesetzt. Bei ordinalskalierten oder nicht normalverteilten intervallskalierten Variablen wurde der Mann-Whitney-U-Test genutzt. Wenn alle beteiligten Variablen kategorial waren, wurde außerdem der $X^{2}$-Test nach Pearson verwendet. Sofern die erwarteten Zellhäu- figkeiten $\leq 5$ waren, wurde stattdessen der Exakte Test nach Fisher verwendet. Für Korrelationsanalysen wurde die Rangkorrelation nach Spearman genutzt, da die korrelierten Variablen nicht normalverteilt waren. Das Signifikanzniveau wurde für alle statistischen Verfahren auf 5 \% festgesetzt. Um der Alphafehler-Kumulierung entgegenzuwirken, ist bei multiplen Mittelwertvergleichen die Bonferroni-Korrektur verwendet worden. Alle Analysen wurden mit dem Statistik- und Analyse-Programm IBM SPSS Statistics 24, IBM, Armonk, USA durchgeführt.

\section{Ergebnisse}

\section{Soziodemografische und medizinische Daten}

Die Studienteilnehmer hatten ein durchschnittliches Alter von 37,3 \pm 15,6 Jahren (Median 33 Jahre, Range 19-71 Jahre), darunter 42 Frauen und 42 Männer. Die guten Schläfer bestanden zu 47,2\% aus weiblichen und zu 52,8\% aus männlichen Probanden, die schlechten Schläfer zu 54,8\% aus weiblichen und zu 45,2\% aus männlichen Probanden, was einer vergleichbaren Verteilung der Geschlechter in den untersuchten Gruppen entsprach $\left(p_{x}{ }^{2}=0,498\right)$. Auch hinsichtlich der anderen untersuchten soziodemografischen und medizinischen Daten wurden keine signifikanten Unterschiede zwischen den beiden Gruppen gefunden. - Tab. 1 zeigt die Daten der deskriptiven Statistik beider Gruppen und der Gesamtstichprobe. Die jeweiligen absoluten und prozentualen Anzahlen des Tätigkeitsprofils und Tabakkonsums beider Gruppen und der Gesamtstichprobe sind in $>$ Tab. $\mathbf{2}$ dargestellt.

Der durchschnittliche BMI der Studienteilnehmer beider Gruppen lag bei $24,55 \pm 4,02 \mathrm{~kg} / \mathrm{m}^{2}$, wobei 52 (61,9\%) nach der geschlechts- und altersunabhängigen WHO-Klassifikation [13] normalgewichtig, 21 (25,0\%) präadipös und 10 (11,9\%) adipös waren. Ein Proband (1,2\%) war untergewichtig. Ein erhöhtes THV (Männer $a b \geq 0,90$; Frauen $a b \geq 0,85$ [14]) hatten allerdings durchschnittlich die männlichen Probanden beider Gruppen (gute Schläfer 0,94 0,09; schlechte Schläfer 0,95 $\pm 0,07 ; p=0,519$ ). Auch die weiblichen Probanden der schlechten Schläfer hatten ein erhöhtes THV $(0,85 \pm 0,11)$, wohingegen die weiblichen Probanden der guten Schläfer ein unauffälliges THV hatten $(0,82 \pm 0,08 ; p=0,359)$.

Um die Packungsjahre (py) zu berechnen, wurden die täglich konsumierten Zigarettenpackungen mit der Anzahl der Raucherjahre multipliziert. Während die 12 aktuellen oder ehemaligen Raucher der guten Schläfer (23,1\%) eine durchschnittliche Dosis von 10,5 py $\pm 9,5$ py rauchten, war es bei den 8 aktuellen oder ehemaligen Rauchern der schlechten Schläfer (26,6\%) eine durchschnittliche Dosis von 17,5 py $\pm 12,9$ py $(p=0,198)$.

\section{Schlafqualität}

- Tab. 3 gibt die erreichten durchschnittlichen Gesamtpunktzahlen und Komponentenpunktzahlen im PSQI mit Standardabweichungen sowie die jeweiligen Mediane und Spannweiten beider Gruppen und der Gesamtstichprobe wieder.

Die guten Schläfer benötigten im Mittel 12:13 min \pm 07:55 min, um nachts einzuschlafen (Median $10 \mathrm{~min}$, Range 2$45 \mathrm{~min}$ ). Die tatsächliche Schlafdauer betrug 07:17 h $\pm 00: 50 \mathrm{~h}$ 
- Tab. 1 Vergleich der soziodemografischen und medizinischen Daten beider Gruppen.

\begin{tabular}{|c|c|c|c|c|}
\hline & $\begin{array}{l}\text { Gute Schläfer } \\
(n=53)\end{array}$ & $\begin{array}{l}\text { Schlechte Schläfer } \\
(\mathrm{n}=31)\end{array}$ & $\begin{array}{l}\text { Gesamt } \\
(n=84)\end{array}$ & \\
\hline & $\begin{array}{l}\text { MW } \pm \text { SD } \\
\text { Median (Range) }\end{array}$ & $\begin{array}{l}\text { MW } \pm \text { SD } \\
\text { Median (Range) }\end{array}$ & $\begin{array}{l}\text { MW } \pm \text { SD } \\
\text { Median (Range) }\end{array}$ & p-Wert \\
\hline $\begin{array}{l}\text { Alter } \\
\text { [Jahre] }\end{array}$ & $\begin{array}{l}35,2 \pm 14,3 \\
29(19-64)\end{array}$ & $\begin{array}{l}40,9 \pm 17,2 \\
47(19-71)\end{array}$ & $\begin{array}{l}37,3 \pm 15,6 \\
33(19-71)\end{array}$ & 0,195 \\
\hline $\begin{array}{l}\mathrm{BMI} \\
{\left[\mathrm{kg} / \mathrm{m}^{2}\right]}\end{array}$ & $\begin{array}{l}24,37 \pm 3,94 \\
23,46(17,63-38,57)\end{array}$ & $\begin{array}{l}24,84 \pm 4,20 \\
24,13(19,33-34,36)\end{array}$ & $\begin{array}{l}24,55 \pm 4,02 \\
23,89(17,63-38,57)\end{array}$ & 0,680 \\
\hline THV & $\begin{array}{l}0,88 \pm 0,11 \\
0,89(0,68-1,29)\end{array}$ & $\begin{array}{l}0,89 \pm 0,11 \\
0,88(0,70-1,20)\end{array}$ & $\begin{array}{l}0,89 \pm 0,11 \\
0,89(0,68-1,29)\end{array}$ & 0,637 \\
\hline $\begin{array}{l}\text { RR sys } \\
{[\mathrm{mmHg}]}\end{array}$ & $\begin{array}{l}125,9 \pm 10,8 \\
125(90-158)\end{array}$ & $\begin{array}{l}124,4 \pm 15,6 \\
121(99-167)\end{array}$ & $\begin{array}{l}125,3 \pm 12,7 \\
124(90-167)\end{array}$ & 0,164 \\
\hline $\begin{array}{l}\text { RR dias } \\
{[\mathrm{mmHg}]}\end{array}$ & $\begin{array}{l}79,3 \pm 8,7 \\
80,5(60-105)\end{array}$ & $\begin{array}{l}78,8 \pm 11,0 \\
78(57-102)\end{array}$ & $\begin{array}{l}79,1 \pm 9,6 \\
79(57-105)\end{array}$ & 0,415 \\
\hline $\begin{array}{l}\text { Sport } \\
\text { [Mal/Woche] }\end{array}$ & $\begin{array}{l}2,3 \pm 1,9 \\
2(0-6)\end{array}$ & $\begin{array}{l}1,8 \pm 2,0 \\
1(0-8)\end{array}$ & $\begin{array}{l}2,1 \pm 2,0 \\
2(0-8)\end{array}$ & 0,251 \\
\hline $\begin{array}{l}\text { Sport } \\
\text { [Jahre] }\end{array}$ & $\begin{array}{l}12,0 \pm 12,2 \\
10(0-45)\end{array}$ & $\begin{array}{l}8,7 \pm 8,9 \\
6(0-30)\end{array}$ & $\begin{array}{l}10,8 \pm 11,1 \\
10(0-45)\end{array}$ & 0,357 \\
\hline
\end{tabular}

p-Wert: Zweistichproben-t-Test bzw. Mann-Whitney-U-Test. BMI: Body-Mass-Index, THV: Taille-Hüft-Verhältnis, RR sys: systolischer Blutdruck, RR dias: diastolischer Blutdruck.

- Tab. 2 Vergleich weiterer soziodemografischer Angaben beider Gruppen.

\begin{tabular}{|c|c|c|c|c|c|}
\hline & & Gute Schläfer & Schlechte Schläfer & Gesamt & \\
\hline & & Anzahl (\%) & & & p-Wert \\
\hline \multirow[t]{5}{*}{ Tätigkeit } & vorwiegend geistig & $40(75,5)$ & $23(74,2)$ & $63(75,0)$ & \multirow{5}{*}{0,424} \\
\hline & vorwiegend körperlich & $5(9,4)$ & $1(3,2)$ & $6(7,1)$ & \\
\hline & körperlich und geistig & $8(15,1)$ & $6(19,4)$ & $14(16,7)$ & \\
\hline & Rentner & $0(0,0)$ & $1(3,2)$ & $1(1,2)$ & \\
\hline & ehemalig & $8(15,4)$ & $7(23,3)$ & $15(18,3)$ & \\
\hline \multirow[t]{2}{*}{ Raucherstatus } & aktuell & $4(7,7)$ & $1(3,3)$ & $5(6,1)$ & \multirow{2}{*}{0,596} \\
\hline & Nichtraucher & $40(76,9)$ & $22(73,3)$ & $62(75,6)$ & \\
\hline
\end{tabular}

und die Schlafeffizienz im PSQI (für Details > Tab. 3 und [15]) war im Median > 85 \% (entspricht 0 Punkten). Demgegenüber brauchten die schlechten Schläfer durchschnittlich 35:00 min \pm 42:04 min, um nachts einzuschlafen (Median 23 min, Range 2$240 \mathrm{~min}$ ), schliefen tatsächlich 05:41 h \pm 01:03 h und die Schlafeffizienz im PSQI lag im Median bei 75-84\% (entspricht 1 Punkt). Ins Bett gingen die guten Schläfer im Median um 22:30 Uhr, die schlechten Schläfer um 22:00 Uhr.

\section{Tagesschläfrigkeit}

Zwar erreichten die schlechten Schläfer leicht höhere Punktzahlen in der ESS als die guten Schläfer ( $\triangleright$ Tab. 4), es ergab sich jedoch kein signifikanter Unterschied zwischen beiden Gruppen $(p=0,113)$.

Auch unter Verwendung des Cut-off-Werts für überhöhte Tagesschläfrigkeit von > 10 Punkten [17-20] wurde kein signifikanter Unterschied gefunden. Hier lag eine vergleichbare Verteilung vor $\left(\mathrm{p}_{\mathrm{x}}{ }^{2}=0,331\right)(\triangleright$ Tab. 5). 
- Tab. 3 Erzielte Gesamtpunktzahlen und Komponentenpunktzahlen im Pittsburgh Sleep Quality Index (PSQI) beider Gruppen.

\begin{tabular}{|c|c|c|c|}
\hline & $\begin{array}{l}\text { Gute Schläfer } \\
(n=53)\end{array}$ & $\begin{array}{l}\text { Schlechte } \\
\text { Schläfer } \\
\text { (n=31) }\end{array}$ & $\begin{array}{l}\text { Gesamt } \\
(n=84)\end{array}$ \\
\hline & $\begin{array}{l}\text { MW } \pm \text { SD } \\
\text { Median } \\
\text { (Range) }\end{array}$ & $\begin{array}{l}\text { MW } \pm \text { SD } \\
\text { Median } \\
\text { (Range) }\end{array}$ & $\begin{array}{l}\text { MW } \pm \text { SD } \\
\text { Median } \\
\text { (Range) }\end{array}$ \\
\hline $\begin{array}{l}\text { Gesamtpunktzahl } \\
\text { PSQI }\end{array}$ & $\begin{array}{l}3,5 \pm 1,3 \\
4(0-5)\end{array}$ & $\begin{array}{l}9,0 \pm 2,6 \\
8(6-14)\end{array}$ & $\begin{array}{l}5,5 \pm 3,3 \\
5(0-14)\end{array}$ \\
\hline $\begin{array}{l}\text { Subjektive } \\
\text { Schlafqualität }\end{array}$ & $\begin{array}{l}0,83 \pm 0,47 \\
1(0-2)\end{array}$ & $\begin{array}{l}1,61 \pm 0,56 \\
2(1-3)\end{array}$ & $\begin{array}{l}1,12 \pm 0,63 \\
1(0-3)\end{array}$ \\
\hline Schlaflatenz & $\begin{array}{l}0,58 \pm 0,50 \\
1(0-1)\end{array}$ & $\begin{array}{l}1,58 \pm 0,93 \\
1(0-3)\end{array}$ & $\begin{array}{l}0,95 \pm 0,84 \\
1(0-3)\end{array}$ \\
\hline Schlafdauer & $\begin{array}{l}0,32 \pm 0,51 \\
0(0-2)\end{array}$ & $\begin{array}{l}1,58 \pm 0,96 \\
2(0-3)\end{array}$ & $\begin{array}{l}0,79 \pm 0,94 \\
1(0-3)\end{array}$ \\
\hline Schlafeffizienz & $\begin{array}{l}0,13 \pm 0,40 \\
0(0-2)\end{array}$ & $\begin{array}{l}1,29 \pm 1,11 \\
1(0-3)\end{array}$ & $\begin{array}{l}0,56 \pm 0,93 \\
0(0-3)\end{array}$ \\
\hline Schlafstörungen & $\begin{array}{l}0,92 \pm 0,34 \\
1(0-2)\end{array}$ & $\begin{array}{l}1,35 \pm 0,49 \\
1(1-2)\end{array}$ & $\begin{array}{l}1,08 \pm 0,45 \\
1(0-2)\end{array}$ \\
\hline Schlafmittelkonsum & $\begin{array}{l}0 \pm 0 \\
0(0-0)\end{array}$ & $\begin{array}{l}0,26 \pm 0,73 \\
0(0-3)\end{array}$ & $\begin{array}{l}0,10 \pm 0,46 \\
0(0-3)\end{array}$ \\
\hline Tagesschläfrigkeit & $\begin{array}{l}0,72 \pm 0,61 \\
1(0-2)\end{array}$ & $\begin{array}{l}1,35 \pm 0,84 \\
1(0-3)\end{array}$ & $\begin{array}{l}0,95 \pm 0,76 \\
1(0-3)\end{array}$ \\
\hline
\end{tabular}

- Tab.4 Erzielte Gesamtpunktzahlen beider Gruppen in der Epworth Sleepiness Scale (ESS).

\begin{tabular}{|l|l|l|l|l|}
\hline & \multicolumn{3}{|c|}{ Gesamtpunktzahl ESS } \\
\hline & MW \pm SD & Median & Range & p-Wert \\
\hline \begin{tabular}{l|l|l|}
\hline \\
Gute Schläfer $(n=53)$
\end{tabular} & $7,1 \pm 3,3$ & 7 & $1-14$ & \\
\hline $\begin{array}{l}\text { Schlechte Schläfer } \\
(n=31)\end{array}$ & $8,8 \pm 4,8$ & 8 & $0-20$ & 0,113 \\
\hline $\begin{array}{l}\text { Gesamt }(n=84) \\
\text { p-Wert: Mann-Whitney-U-Test. }\end{array}$ & & $0-20$ & \\
\hline
\end{tabular}

Während 9 gute Schläfer (17,0\%) einen erhöhten Wert erreichten, waren es 8 schlechte Schläfer (25,8\%).

\section{Zusammenhänge zwischen subjektiver Schlafqualität und individueller Tagesschläfrigkeit}

Bei der Korrelationsanalyse der Parameter aus den beiden Fragebögen zur Schlafqualität und Tagesschläfrigkeit wurde festgestellt, dass zwischen der Gesamtpunktzahl des PSQI und der Gesamtpunktzahl der ESS kein signifikanter Zusammenhang besteht ( $p=0,094 ;$ - Tab. 6).
Bis auf die Komponente Tagesschläfrigkeit im PSQI ( $r=0,365$, $p<0,001)$ korrelierten auch die einzelnen Komponentenpunktzahlen des PSQI nicht signifikant mit der Gesamtpunktzahl der ESS.

\section{Arbeitsbezogene Beanspruchungsfolgen}

Ein Proband aus der Gruppe der guten Schläfer und ein Proband der schlechten Schläfer füllten die IS nicht ordnungsgemäß aus, weswegen sich dieser Teil der Auswertung nur auf 82 Probanden bezieht (gute Schläfer $n=52$, schlechte Schläfer $n=30$ ). Es lagen signifikante Unterschiede zwischen beiden Gruppen in den Subskalen $\mathrm{KI}(p=0,004)$ und $\mathrm{EI}(p=0,009)$ sowie im GI $(p=0,003)$ vor. Die schlechten Schläfer erreichten dabei höhere Punktzahlen als die guten Schläfer ( $\vee$ Tab. 7).

Transformiert man die Rohwertpunkte mithilfe der von Mohr et al. [21, 23] empfohlenen altersspezifischen Normierung, so zeigen sich für alle drei Skalen ebenfalls signifikant unterschiedliche Verteilungen (KI: $p=0,019$, El: $p=0,033, G I: p=0,016$; Tab. 8).

Die schlechten Schläfer wurden prozentual in allen drei Skalen deutlich häufiger als „überdurchschnittlich“ eingestuft, was für eine höhere Ausprägung an Irritation spricht. Dagegen waren in der Einstufung „unterdurchschnittlich“ prozentual deutlich mehr gute Schläfer vertreten.

\section{Zusammenhänge zwischen arbeitsbezogenen Beanspruchungsfolgen und subjektiver Schlafqualität bzw. Tagesschläfrigkeit}

Weitere Korrelationsanalysen wurden durchgeführt, um die Zusammenhänge zwischen arbeitsbezogenen Beanspruchungsfolgen und subjektiver Schlafqualität bzw. Tagesschläfrigkeit weiter zu untersuchen. Zwischen der Gesamtpunktzahl des PSQI und den jeweiligen Punktzahlen von KI, El und GI wurden signifikante positive Korrelationen gefunden (KI: $r=0,386$, El: $r=0,409$, Gl: $r=0,427$, jeweiliges $p<0,001)$. Je niedriger die Schlafqualität war (= höhere Gesamtpunktzahl im PSQI), desto stärker ausgeprägt waren die verschiedenen Formen der Irritation. Bei den einzelnen Komponentenpunktzahlen korrelierten alle Komponenten (bis auf die Komponente Schlafeffizienz) mit mindestens einer der 3 Skalen der IS ebenfalls signifikant ( Tab.9).

Auch bei der Korrelation der Gesamtpunktzahl der ESS mit der Punktzahl der El wurde eine signifikante positive Korrelation nachgewiesen $(r=0,299, p=0,006)$. Mit zunehmender Tagesschläfrigkeit stieg der Wert für die Emotionale Irritation. Andererseits wurden bei der Korrelation der Gesamtpunktzahl der ESS mit der Punktzahl von KI und GI keine signifikanten Zusammenhänge gefunden (KI: $p=0,355, G l: p=0,070)$.

\section{Diskussion}

Erholsamer Schlaf nimmt eine wichtige Rolle für Gesundheit, subjektives Wohlbefinden und Lebensqualität ein [7]. Permanente psychische Belastungen am Arbeitsplatz wie Aufgabenhäufung, Arbeit unter Zeitdruck, Tätigkeit mit hoher Verantwortung für das Leben anderer, ständige Erreichbarkeit, aber auch Angst um den Arbeitsplatz und Mobbing können Auswirkungen auf die Schlafqualität und -quantität, u. a. Ein- und Durchschlafprobleme, 
- Tab. 5 Einteilung der beiden Gruppen nach Cut-off-Wert der Epworth Sleepiness Scale (ESS).

\begin{tabular}{|l|l|l|l|l|}
\hline & Gute Schläfer & Schlechte Schläfer & Gesamt \\
\hline & Anzahl (\%) & & \\
\hline & & & \\
\hline
\end{tabular}

- Tab. 6 Korrelationen der Gesamtpunktzahl und der Komponentenpunktzahlen des Pittsburgh Sleep Quality Index (PSQI) mit der Gesamtpunktzahl der Epworth Sleepiness Scale (ESS).

\begin{tabular}{|l|l|l|l|l|l|l|l|l|l|}
\hline & \multicolumn{9}{|c|}{ PSQI-Komponenten } \\
\hline & $\begin{array}{l}\text { Gesamt- } \\
\text { punktzahl } \\
\text { PSQI }\end{array}$ & $\begin{array}{l}\text { Subjektive } \\
\text { Schlafqualität }\end{array}$ & $\begin{array}{l}\text { Schlaf- } \\
\text { latenz }\end{array}$ & $\begin{array}{l}\text { Schlaf- } \\
\text { dauer }\end{array}$ & $\begin{array}{l}\text { Schlaf- } \\
\text { effizienz }\end{array}$ & $\begin{array}{l}\text { Schlaf- } \\
\text { störungen }\end{array}$ & $\begin{array}{l}\text { Schlafmit- } \\
\text { telkonsum }\end{array}$ \\
\hline & & & & & & & \\
\hline
\end{tabular}

- Tab.7 Erzielte Gesamtpunktzahlen beider Gruppen in den Subskalen Kognitive Irritation (KI) und Emotionale Irritation (EI) sowie im Gesamtindex (GI) der Irritations-Skala zur Erfassung arbeitsbezogener Beanspruchungsfolgen (IS).

\begin{tabular}{|l|c|c|c|}
\hline & Gesamtpunktzahl KI & & Range \\
\hline & MW \pm SD & Median & \\
\hline KI & & 6 & $3-21$ \\
\hline Gute Schläfer $(n=52)$ & $7,9 \pm 4,4$ & $3-20$ \\
\hline Schlechte Schläfer $(n=30)$ & $11,0 \pm 4,9$ & $3-21$ \\
\hline Gesamt $(n=82)$ & $9,0 \pm 4,8$ & $\mathbf{0 , 0 0 4}$ \\
\hline El & & 8 & $5-25$ \\
\hline Gute Schläfer $(n=52)$ & $11,1 \pm 5,2$ & $5-32$ \\
\hline Schlechte Schläfer $(n=30)$ & $15,6 \pm 8,1$ & $5-32$ \\
\hline Gesamt $(n=82)$ & $12,7 \pm 6,7$ & 14 & $\mathbf{0 , 0 0 9}$ \\
\hline Gl & & 11 & $8-36$ \\
\hline Gute Schläfer $(n=52)$ & $19,0 \pm 7,5$ & $10-52$ \\
\hline Schlechte Schläfer $(n=30)$ & $26,6 \pm 11,7$ & $8-52$ \\
\hline Gesamt $(n=82)$ & $21,8 \pm 9,9$ & 24 & $\mathbf{0 , 0 0 3}$ \\
\hline p-Wert: Mann-Whitney-U-Test. $p<0,05$ fett dargestellt. & & 21 & \\
\hline
\end{tabular}

haben [24]. Da Schlaf nicht bewusst erlebt wird, kann seine Qualität subjektiv nur im Nachhinein bewertet werden [25]. In der vorliegenden Studie wurde hierfür der PSQI verwendet, ein standardisiertes Verfahren aus der Schlafmedizin, das zwischen guten und schlechten Schläfern unterscheidet. Dies wurde genutzt, um die Gesamtstichprobe in zwei Gruppen aufzuteilen. 
- Tab. 8 Einteilung beider Gruppen nach altersspezifischen Normwerten der Subskalen Kognitive Irritation (KI) und Emotionale Irritation (EI) sowie des Gesamtindexes (GI) der Irritations-Skala zur Erfassung arbeitsbezogener Beanspruchungsfolgen (IS).

\begin{tabular}{|c|c|c|c|c|c|}
\hline & & Gute Schläfer & Schlechte Schläfer & Gesamt & \\
\hline & & \multicolumn{3}{|l|}{ Anzahl (\%) } & p-Wert \\
\hline \multirow{3}{*}{ KI } & unterdurchschnittlich & $22(42,3)$ & $5(16,7)$ & $27(32,9)$ & \multirow{3}{*}{0,019} \\
\hline & durchschnittlich & $24(46,2)$ & $15(50,0)$ & $39(47,6)$ & \\
\hline & überdurchschnittlich & $6(11,5)$ & $10(33,3)$ & $16(19,5)$ & \\
\hline \multirow{3}{*}{$\mathrm{EI}$} & unterdurchschnittlich & $21(40,4)$ & $5(16,7)$ & $26(31,7)$ & \multirow{3}{*}{0,033} \\
\hline & durchschnittlich & $25(48,1)$ & $16(53,3)$ & $41(50,0)$ & \\
\hline & überdurchschnittlich & $6(11,5)$ & $9(30,0)$ & $15(18,3)$ & \\
\hline \multirow{3}{*}{ GI } & unterdurchschnittlich & $18(34,6)$ & $7(23,3)$ & $25(30,5)$ & \multirow{3}{*}{0,016} \\
\hline & durchschnittlich & $31(59,6)$ & $14(46,7)$ & $45(54,9)$ & \\
\hline & überdurchschnittlich & $3(5,8)$ & $9(30,0)$ & $12(14,6)$ & \\
\hline
\end{tabular}

p-Wert: Exakter Test nach Fisher, da erwartete Zellhäufigkeiten $\leq 5 . \mathrm{p}<0,05$ fett dargestellt.

Dabei wurde auf die Assoziation mit den arbeitsbezogenen Beanspruchungsfolgen geachtet.

Bezüglich der untersuchten soziodemografischen und medizinischen Daten unterschieden sich beide Gruppen nicht signifikant. Dieser Umstand stellt für die weiteren Analysen eine ideale Voraussetzung dar, da somit vergleichbare Grundvoraussetzungen in beiden Gruppen bestanden. Die nicht vorhandenen Unterschiede sind auffällig, da einige dieser Faktoren zum Teil mit schlechter Schlafqualität und kurzer Schlafdauer assoziiert sind, beispielsweise hoher Blutdruck [26] und Adipositas [27, 28].

Die arbeitsbezogenen Beanspruchungsfolgen, die über KI und El sowie den GI der IS analysiert wurden, unterschieden sich signifikant zwischen den Gruppen mit unterschiedlicher Schlafqualität, wobei die schlechten Schläfer im Mittel höhere Werte in allen 3 Skalen erzielten und die arbeitsbezogenen Beanspruchungsfolgen infolgedessen häufiger als überdurchschnittlich eingestuft wurden. Auch in den Korrelationsanalysen zeigten sich signifikant positive Zusammenhänge von KI, El und GI mit der subjektiven Schlafqualität im PSQI. Zusätzlich fand sich eine signifikant positive Korrelation zwischen El und der Tagesschläfrigkeit in der ESS.

Da es sich um eine Querschnittsuntersuchung handelt, können keine Rückschlüsse auf die Kausalität gezogen werden. Es ist sowohl möglich, dass subjektive Schlafqualität Einfluss auf psychische Beanspruchungsfolgen hatte als auch dass sich psychische Beanspruchungsfolgen auf subjektive Schlafqualität auswirkten. Wahrscheinlich ist ein bidirektionaler Zusammenhang, da gestörter Schlaf auf der einen Seite Symptom unterschiedlicher psychischer Erkrankungen und Zustände ist [12], auf der anderen Seite aber auch zu Burnout [29, 30] und psychischen Erkrankungen [31] führt und Irritation als Vorläufer psychischer Befindensbeeinträchtigung aufgefasst werden kann [22].

Berufliche Unsicherheit trägt zu einem bedeutenden Anteil zur Gesamtheit der psychischen Belastung bei [32]. Beschäftigte, die ihre Entlassungsgefährdung als wahrscheinlich einschätzen, haben eine höhere Prävalenz für Insomnie als Beschäftigte, die ihr Arbeitsverhältnis als sicher betrachten [33]. Verschiedene Studien konnten einen Zusammenhang zwischen Schlafqualität und arbeitsbedingtem psychosozialem Stress nachweisen [8-11].

Ständige Erreichbarkeit in der modernen Arbeitswelt kann Vor-, aber auch Nachteile für den Gesundheitszustand der Arbeitnehmer in sich bergen. Eine positive Beanspruchungsfolge von ständiger Erreichbarkeit ist die flexible Arbeitsortwahl bei nicht an die Arbeitsstätte gebundenen Arbeitsanforderungen. Die erweiterte Verfügbarkeit kann aber auch zusätzliche Arbeitsbelastungen außerhalb der regulären Arbeitszeit und Fragmentierung der einzelnen Lebensbereiche zur Folge haben, was Auswirkungen auf Beanspruchung und Erholung hat. Es kann zu Reduzierung oder sogar Wegfall von Erholungszeit kommen und zu Verringerung der Distanzierungsfähigkeit [34]. In der modernen Gesellschaft geht die Entwicklung immer mehr in Richtung ständige Erreichbarkeit, Flexibilität und Mobilität [35], weshalb davon auszugehen ist, dass ständige Erreichbarkeit ein beitragendes Element für höhere Punktzahlen in der IS und somit höheren Beanspruchungsfolgen war. Viele Abläufe müssen zeitlich, örtlich und situativ an die ständige Erreichbarkeit angepasst werden [34] und bereits die Perspektive kontaktiert zu werden, führt zu einem Anstieg von Reizbarkeit, Verringerung sozialer sowie häuslicher Aktivitäten und Verschlechterung der Stimmung [36]. Auf diese Weise kann nachts der Schlaf quantitativ und qualitativ beeinträchtigt werden [34, 37]. Insbesondere Führungskräfte mit hohem Verantwortungsdruck und hohem Aufkommen an Arbeitsaufgaben empfinden ständige Erreichbarkeit als besonders beanspruchend und berichten über Ein- und Durchschlafprobleme [24]. Sofern aus der Beeinträchtigung des Schlafes Tagesschläfrigkeit resultiert, scheint diese insbesondere mit sozialen Stressoren (Abbild der El) und eher nicht mit tätigkeitsspezifischen Stressoren (Abbild der KI) assoziiert zu sein.

Eine vorstellbare Verzerrung des beschriebenen Zusammenhangs ist, dass ein Proband mit hohen arbeitsbezogenen Beanspruchungsfolgen bzw. schlechter subjektiver Schlafqualität mög- 


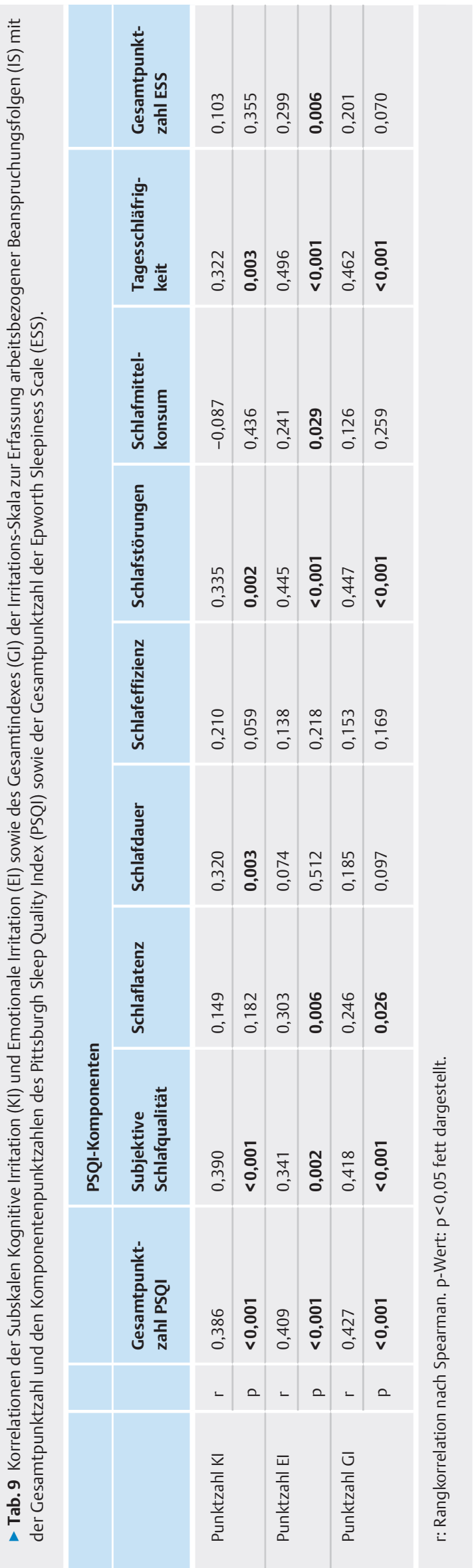

licherweise bereits eine negative pessimistische Grundeinstellung hat und den jeweils anderen Faktor bewusst oder unbewusst schlechter beurteilt, als er tatsächlich ist. Dagegen hat ein Proband mit geringen arbeitsbezogenen Beanspruchungsfolgen bzw. guter subjektiver Schlafqualität gegebenenfalls eine positive optimistische Grundeinstellung und schätzt den jeweils anderen Faktor bewusst oder unbewusst besser ein, als er wirklich ist.

Limitationen dieser Studie sind ihr querschnittliches Design und die kleine Stichprobengröße. Außerdem hängen die Ergebnisse von der Selbstbeurteilung der Teilnehmer ab, da es hier nur um die subjektive Einschätzung durch den Probanden geht. Weitere Untersuchungen mit größeren Stichproben und prospektivem Design werden benötigt, um die gewonnenen Erkenntnisse weiter zu belegen. In einer weiterführenden Studie könnte auch die Art der Schlafprobleme genauer untersucht werden, zum Beispiel mit einem geeigneten Insomnie- und Schlafapnoefragebogen.

Zusammenfassend liefern die Ergebnisse dieser Studie weitere Indizien dafür, dass wichtige Wechselbeziehungen zwischen subjektiver Schlafqualität und arbeitsbezogenen Beanspruchungsfolgen bestehen.

\section{KERNAUSSAGEN}

- Es bestand ein Zusammenhang zwischen arbeitsbezogenen Beanspruchungsfolgen und subjektiver Schlafqualität.

- Schlechte Schläfer hatten eine höhere arbeitsbezogene Beanspruchung als gute Schläfer.

- Es bestand ein Zusammenhang zwischen individueller Tagesschläfrigkeit und arbeitsbezogenen Beanspruchungsfolgen in Form von Emotionaler Irritation, aber nicht in Form von Kognitiver Irritation sowie Gesamtirritation.

\section{Finanzielle Unterstützung}

Diese Studie wurde finanziell nicht unterstützt.

Interessenkonflikt

Die Autoren geben an, dass kein Interessenkonflikt besteht.

Literatur

[1] Bauer J. Psychische Belastungen am Arbeitsplatz. Rechtliche Rahmenbedingungen. Zbl Arbeitsmed 2016; 66: 47-49. doi:10.1007/s40664-0150071-7

[2] Grobe T, Steinmann S, Gerr ]. AQUA - Institut für angewandte Qualitätsförderung und Forschung im Gesundheitswesen $\mathrm{GmbH}$. Gesundheitsreport 2017. Weitere Auswertungen zu Arbeitsunfähigkeiten. Hamburg: Techniker Krankenkasse. 2017

[3] Deutsches Institut für Normung. DIN EN ISO 10075-1. Ergonomische Grundlagen bezüglich psychischer Arbeitsbelastung - Teil 1: Allgemeine Konzepte und Begriffe (ISO/DIS 10075-1:2015). Berlin: Beuth. 2015 
[4] Gemeinsame Deutsche Arbeitsschutzstrategie. Leitlinie Beratung und Überwachung bei psychischer Belastung am Arbeitsplatz. Berlin: Geschäftsstelle der Nationalen Arbeitsschutzkonferenz c/o Bundesanstalt für Arbeitsschutz und Arbeitsmedizin. 2015

[5] Joiko K, Schmauder M, Wolff G. Psychische Belastung und Beanspruchung im Berufsleben. Erkennen - Gestalten. Dortmund-Dorstfeld: Bundesanstalt für Arbeitsschutz und Arbeitsmedizin (BAuA). 2010

[6] Selye H. The Stress of Life. New York: McGraw-Hill; 1956

[7] Happe S. Schlaf und seine Störungen im Alter. Bundesgesundheitsblatt Gesundheitsforschung Gesundheitsschutz 2011; 54: 1311-1318. doi:10.1007/s00103-011-1371-5

[8] Kalimo R, Tenkanen L, Härmä M et al. Job stress and sleep disorders: findings from the Helsinki Heart Study. Stress Health 2000; 16: 65-75. doi:10.1002/(SICI)1099-1700(200003)16:2<65: AID-SMI834>3.0.CO;2-8

[9] Åkerstedt T, Fredlund P, Gillberg $M$ et al. Work load and work hours in relation to disturbed sleep and fatigue in a large representative sample. J Psychosom Res 2002; 53: 585-588. doi:10.1016/S00223999(02)00447-6

[10] De Lange AH, Kompier MAJ, Taris TW et al. A hard day's night: a longitudinal study on the relationships among job demands and job control, sleep quality and fatigue. J Sleep Res 2009; 18: 374-383. doi:10.1111/ j.1365-2869.2009.00735.x

[11] Åkerstedt T, Nordin M, Alfredsson L et al. Predicting changes in sleep complaints from baseline values and changes in work demands, work control, and work preoccupation - The WOLF-project. Sleep Med 2012; 13: 73-80. doi:10.1016/j.sleep.2011.04.015

[12] Krystal AD. Psychiatric Disorders and Sleep. Neurol Clin 2012; 30: 13891413. doi:10.1016/j.ncl.2012.08.018

[13] World Health Organization (WHO). Obesity: Preventing And Managing The Global Epidemic. Report of a WHO Consultation. Technical Report Series 894. Genf: World Health Organization (WHO). 2000

[14] World Health Organization (WHO). Waist Circumference and Waist-Hip Ratio. Report of a WHO Expert Consultation. Geneva, 8-11 December 2008. Genf: World Health Organization (WHO). 2011

[15] Buysse DJ, Reynolds CF, Monk TH et al. The Pittsburgh Sleep Quality Index: A New Instrument for Psychiatric Practice and Research. Psychiatry Res 1989; 28: 193-213. doi:10.1016/0165-1781(89)90047-4

[16] von Elm E, Altman DG, Egger M et al. Das Strengthening the Reporting of Observational Studies in Epidemiology (STROBE-) Statement. Leitlinien für das Berichten von Beobachtungsstudien. Internist 2008; 49: 688693. doi:10.1007/s00108-008-2138-4

[17] Johns MW. A New Method for Measuring Daytime Sleepiness: The Epworth Sleepiness Scale. Sleep 1991; 14: 540-545. doi:10.1093/sleep/ 14.6.540

[18] Johns MW, Hocking B. Daytime Sleepiness and Sleep Habits of Australian Workers. Sleep 1997; 20: 844-849. doi:10.1093/sleep/20.10.844

[19] Bloch KE, Schoch OD, Zhang JN et al. German Version of the Epworth Sleepiness Scale. Respiration 1999; 66: 440-447. doi:10.1159/ 000029408

[20] Sauter C, Popp R, Danker-Hopfe H et al. Normative values of the German Epworth Sleepiness Scale. Somnologie (Berl) 2007; 11: 272-278. doi:10.1007/s11818-007-0322-8

[21] Mohr G, Rigotti T, Müller A. Irritations-Skala zur Erfassung arbeitsbezogener Beanspruchungsfolgen. Göttingen: Hogrefe; 2007
[22] Müller A, Mohr G, Rigotti T. Differenzielle Aspekte psychischer Beanspruchung aus Sicht der Zielorientierung. Z Differ Diag Psychol 2004; 25 : 213-225. doi:10.1024/0170-1789.25.4.213

[23] Mohr G, Müller A, Rigotti T. Normwerte der Skala Irritation: Zwei Dimensionen psychischer Beanspruchung. Diagnostica 2005; 51: 12-20. doi:10.1026/0012-1924.51.1.12

[24] Minow A, Swart E. Arbeitsbezogene erweiterte Erreichbarkeit. Bedingungen des Beanspruchungsempfindens bei Führungskräften der Sozialund Gesundheitsbranche. Zbl Arbeitsmed 2018. doi:10.1007/s40664018-0285-6

[25] Heitmann J, Cassel W, Ploch T et al. Messung von Schlafdauer und Schlafqualität. Bundesgesundheitsblatt Gesundheitsforschung Gesundheitsschutz 2011; 54: 1276-1283. doi:10.1007/s00103-011-1375-1

[26] Gangwisch JE, Heymsfield SB, Boden-Albala B et al. Short Sleep Duration as a Risk Factor for Hypertension. Analyses of the First National Health and Nutrition Examination Survey. Hypertension 2006; 47: 833-839. doi:10.1161/01.HYP.0000217362.34748.e0

[27] Taheri S, Lin L, Austin D et al. Short Sleep Duration Is Associated with Reduced Leptin, Elevated Ghrelin, and Increased Body Mass Index. PLoS Med 2004; 1: 210-217. doi:10.1371/journal.pmed.0010062

[28] Patel SR, Hu FB. Short Sleep Duration and Weight Gain: A Systematic Review. Obesity (Silver Spring) 2008; 16: 643-53. doi:10.1038/ oby.2007.118

[29] Söderström M, Jeding K, Ekstedt M et al. Insufficient Sleep Predicts Clinical Burnout. J Occup Health Psychol 2012; 17: 175-183. doi:10.1037| a0027518

[30] Elfering A, Kottwitz MU, Tamcan O et al. Impaired sleep predicts onset of low back pain and burnout symptoms: evidence from a three-wave study. Psychol Health Med 2018. doi:10.1080/13548506.2018.1479038

[31] Buysse D], Angst J, Gamma A et al. Prevalence, Course, and Comorbidity of Insomnia and Depression in Young Adults. Sleep 2008; 31: 473-480. doi:10.1093/sleep/31.4.473

[32] Lohmann-Haislah A. Stressreport Deutschland 2012. Psychische Anforderungen, Ressourcen und Befinden. Dortmund/Berlin/Dresden: Bundesanstalt für Arbeitsschutz und Arbeitsmedizin (BAuA). 2012

[33] Marschall ], Hildebrandt S, Sydow H et al. Unter Mitarbeit von Burgart E, Woköck T. Gesundheitsreport 2017. Analyse der Arbeitsunfähigkeitsdaten. Update: Schlafstörungen. Heidelberg: medhochzwei Verlag GmbH; 2017

[34] Hassler M, Rau R, Hupfeld J et al. Unter Mitarbeit von Schuchart U. iga. Report 23. Auswirkungen von ständiger Erreichbarkeit und Präventionsmöglichkeiten. Teil 2: Eine wissenschaftliche Untersuchung zu potenziellen Folgen für Erholung und Gesundheit und Gestaltungsvorschläge für Unternehmen. Berlin: Initiative Gesundheit und Arbeit (iga). 2016

[35] Dettmers ], Vahle-Hinz T, Friedrich $\mathrm{N}$ et al. Entgrenzung der täglichen Arbeitszeit - Beeinträchtigungen durch ständige Erreichbarkeit bei Rufbereitschaft. In: Badura B, Ducki A, Schröder H, et al., Hrsg. FehlzeitenReport 2012. Gesundheit in der flexiblen Arbeitswelt: Chancen nutzen Risiken minimieren. Berlin, Heidelberg: Springer Verlag; 2012: 53-60. doi:10.1007/978-3-642-29201-9_6

[36] Bamberg E, Dettmers ], Funck $\mathrm{H}$ et al. Effects of On-Call Work on WellBeing: Results of a Daily Survey. Appl Psychol Health Well Being 2012; 4: 299-320. doi:10.1111/j.1758-0854.2012.01075.x

[37] Wuyts ], De Valck E, Vandekerckhove $M$ et al. Effects of pre-sleep simulated on-call instructions on subsequent sleep. Biol Psychol 2012; 91 : 383-388. doi:10.1016/j.biopsycho.2012.09.003 